\title{
An explanation for the unusual IMF in Taurus
}

\author{
S. P. Goodwin, A. P. Whitworth, and D. Ward-Thompson \\ Department of Physics \& Astronomy, Cardiff University, PO Box 913, 5 The Parade, Cardiff CF24 3YB, UK \\ Received 4 December 2003 / Accepted 20 February 2004

\begin{abstract}
In comparison with other well studied star formation regions, Taurus is unusual in several respects. (i) Its stellar initial mass function (IMF) peaks at relatively high mass $\left(\sim 0.8 M_{\odot}\right)$, but contains very few stars much more massive than $1 M_{\odot}$, and is relatively deficient in brown dwarfs. (ii) It has a higher binary fraction, particularly at large separations. (iii) Its core mass function is strongly peaked at a few $M_{\odot}$, and the cores have extended envelopes and relatively low levels of turbulence.

We present here the results of an ensemble of hydrodynamic simulations which suggest that the unusual stellar IMF in Taurus is a direct consequence of the unusual properties of the cores there. By following the collapse and fragmentation of cores having properties typical of Taurus, we find that roughly $50 \%$ of the objects formed in a core, predominantly the low-mass ones, are ejected from the core to form a population of low-mass stars and brown dwarfs with a flat mass function. The remaining objects form multiple systems within the core, accreting until their masses approach $1 M_{\odot}$; this produces a population of intermediatemass stars whose mass function peaks at $\sim 0.8 M_{\odot}$. Together these two populations reproduce the IMF in Taurus very well. This demonstrates, for the first time, a direct causal link between the core mass function and the stellar IMF in a star formation region.
\end{abstract}

Key words. stars: formation - ISM: clouds - ISM: structure - methods: numerical

\section{Introduction}

Stars form within dense molecular cores (e.g. André et al. 2000), and the densest and most centrally condensed cores, i.e. those closest to forming stars, are known as prestellar cores (Ward-Thompson et al. 1994). In Ophiuchus (Motte et al. 1998), Serpens (Testi \& Sargent 1998) and Orion (Motte et al. 2001), the mass function of pre-stellar cores is remarkably similar to the IMF for field stars and clustered star formation regions. This suggests that there is a simple mapping from the core mass function into the stellar IMF, with the mean masses of the stars forming within a core being proportional to the core mass. However, the details of how this works are still uncertain.

In this paper we present numerical simulations of core collapse and fragmentation which demonstrate a causal link between the core mass function and the stellar IMF in the Taurus star formation region. We choose to study the Taurus region, because it is nearby and extended on the sky, and has therefore been studied in detail. Molecular-line mapping has yielded estimates of the Taurus core mass function (Onishi et al. 2002), and deep surveys of its stellar content have revealed the Taurus IMF down to the deuterium burning limit (Luhman et al. 2003a).

\section{Observational background}

Determining and explaining the stellar initial mass function (IMF) - i.e. the number of stars, $\mathcal{N}_{M} \mathrm{~d} M$, forming with mass

Send offprint requests to: $\mathrm{S}$. P. Goodwin, e-mail: Simon.Goodwin@astro.cf.ac.uk in the interval $(M, M+\mathrm{d} M)-$ is one of the longest standing problems in the study of star formation.

Salpeter (1955) first investigated the IMF for field stars in the solar neighbourhood and found a power-law fit, $\mathcal{N}_{M} \propto M^{-\alpha}$ with $\alpha \simeq 2.35$, for stars in the mass range $\left(0.4 M_{\odot}<M<\right.$ $10 M_{\odot}$ ). Subsequent work (e.g. Miller \& Scalo 1979; Scalo 1986; Kroupa 2002) has extended the range to both lower and higher masses. In the field, Kroupa (2002) finds that Salpeter's result is still a good approximation for intermediate and high masses $\left(M>0.5 M_{\odot}\right)$, but at lower masses the stellar IMF flattens (i.e. $\alpha$ is smaller). Specifically, $\alpha \sim 1.3 \pm 0.5$ for low-mass stars, $0.08 M_{\odot}<M<0.5 M_{\odot}$; and $\alpha \sim 0.3 \pm 0.7$ for brown dwarfs, $0.01 M_{\odot}<M<0.08 M_{\odot}$.

In individual star formation regions, there appears to be some variance in the stellar IMF, but in regions of clustered star formation this variance is small. For example, Muench et al. (2002) and Luhman et al. (2003b) find stellar IMFs broadly similar to Kroupa's field star IMF in - respectively - Orion and IC 348.

However, in Taurus, which is a region of distributed star formation, the stellar IMF is markedly different. Figure 1 compares the IMF of Taurus (solid-line histogram, adapted from Luhman et al. 2003a) with that of Orion (shaded histogram, adapted from Muench et al. 2002). We note the following points from Fig. 1:

- the Taurus IMF peaks at a higher mass, $\sim 0.8 M_{\odot}$ (as compared with $\sim 0.2 M_{\odot}$ in Orion); 


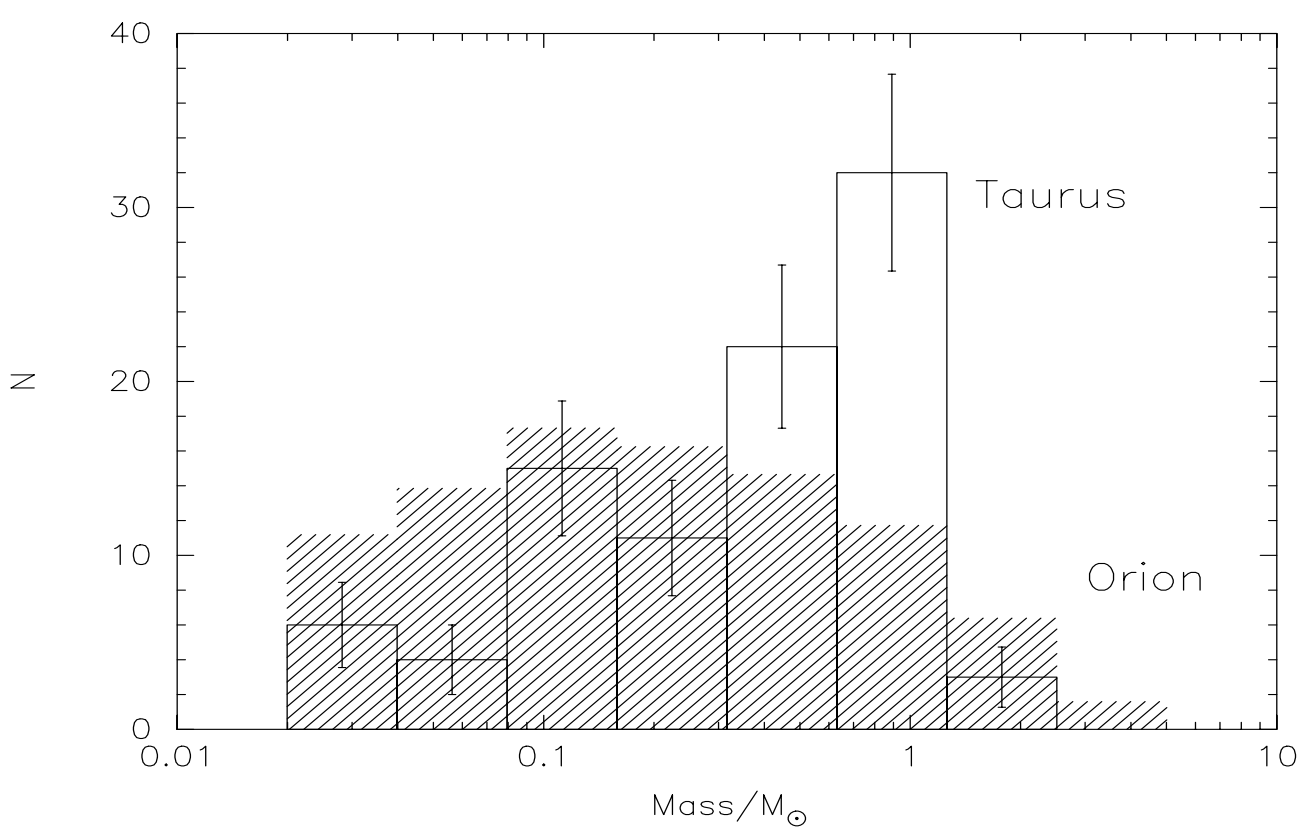

Fig. 1. A plot of number of stars, $N$, versus logarithmic stellar mass, $\log _{10}[M]$, comparing the IMF in Taurus (solid-line histogram, adapted from Luhman et al. 2003a) with that in Orion (shaded histogram, adapted from Muench et al. 2002). The Orion IMF is normalised to have the same number of stars as the Taurus IMF. The two IMFs are clearly different.

- the Taurus IMF contains very few stars more massive than $1 M_{\odot}$ (whereas Orion contains several stars above $10 M_{\odot}$ );

- the Taurus IMF contains many fewer brown dwarfs per star than Orion (less than half as many);

- at the low-mass end the Taurus IMF is approximately flat.

There is further evidence that the pattern of star formation in Taurus is different from that in regions of clustered star formation.

- There is a higher binary fraction in Taurus than in Orion, particularly at large separations;

- the prestellar cores in Taurus (Onishi et al. 2002) have a narrower mass function than those in Orion (Motte et al. 2001) or Ophiuchus (Motte et al. 1998); most cores in Taurus have $M \sim 5 \pm 3 M_{\odot}$;

- the prestellar cores in Taurus are on average more extended and less turbulent than those in Orion and Ophiuchus.

\section{Modelling the collapse and fragmentation of pre-stellar cores in Taurus}

\subsection{Initial conditions and constitutive physics}

In view of the strongly peaked core mass function in Taurus, we consider a single core mass, $M=5 M_{\odot}$. The initial conditions are chosen to mimic the cores observed in Taurus (Ward-Thompson et al. 1994, 1999, 2002; Jijina et al. 1999; Kirk et al. 2003). The core density profile is initially

$\rho=\rho_{\text {kernel }}\left[1+\left(\frac{r}{r_{\text {kernel }}}\right)^{2}\right]^{-2}, r<r_{\text {boundary }}$

with $\rho_{\text {kernel }}=3 \times 10^{-18} \mathrm{~g} \mathrm{~cm}^{-3}$ (corresponding to $n_{\mathrm{H}_{2}} \simeq 7 \times$ $\left.10^{5} \mathrm{~cm}^{-3}\right), r_{\text {kernel }}=5000 \mathrm{AU}$ and $r_{\text {boundary }}=50000 \mathrm{AU}$.
The isothermal sound speed in a core is initially $c_{0}=$ $0.19 \mathrm{~km} \mathrm{~s}^{-1}$ (corresponding to molecular gas at $10 \mathrm{~K}$ ), and so the initial Jeans mass in the centre of a core is $\sim 0.8 M_{\odot}$, and the initial ratio of thermal to gravitational potential energy is $U_{\text {thermal }} /|\Omega|=0.45$. In order to capture the switch from isothermality to adiabaticity which is expected to occur as the density rises above $\rho_{\text {crit }} \sim 10^{-13} \mathrm{~g} \mathrm{~cm}^{-3}$ (e.g. Tohline 1982; Masunaga $\&$ Inutsuka 2000), we use the equation of state

$P=\rho c_{0}^{2}\left[1+\left(\frac{\rho}{\rho_{\text {crit }}}\right)^{2 / 3}\right]$.

A divergence-free Gaussian random velocity field is superimposed on each core to simulate turbulence. The power spectrum of the turbulence is $P(k) \propto k^{-4}$. Fifty realizations of this representative core are treated. The only difference between the different realizations is the random number seed used to generate the turbulent velocity field, and the initial ratio of turbulent to gravitational potential energy, $U_{\text {turbulent }} /|\Omega|$. In order to match the levels of turbulence measured in Taurus, twenty cores have $U_{\text {turbulent }} /|\Omega|=0.05$, twenty cores have $U_{\text {turbulent }} /|\Omega|=0.10$, and ten cores have $U_{\text {turbulent }} /|\Omega|=0.20$.

\subsection{Numerical method}

The simulations are performed with a standard SPH code (e.g. Lucy 1977; Gingold \& Monaghan 1977; Monaghan 1992). For details of the code, see Turner et al. (1995) and Goodwin et al. (2004a,b). It uses variable smoothing lengths $h$, the M 4 smoothing kernel (Monaghan \& Lattanzio 1985), and $\mathcal{N}_{\text {neib }}=50 \pm 5$ neighbours. The artificial viscosity prescription of Lattanzio et al. (1986) is used with $\alpha_{\text {visc }}=1$ and $\beta_{\text {visc }}=2$. An octal tree (Barnes \& Hut 1986) is used to construct neighbour lists and to calculate gravitational accelerations, which are 


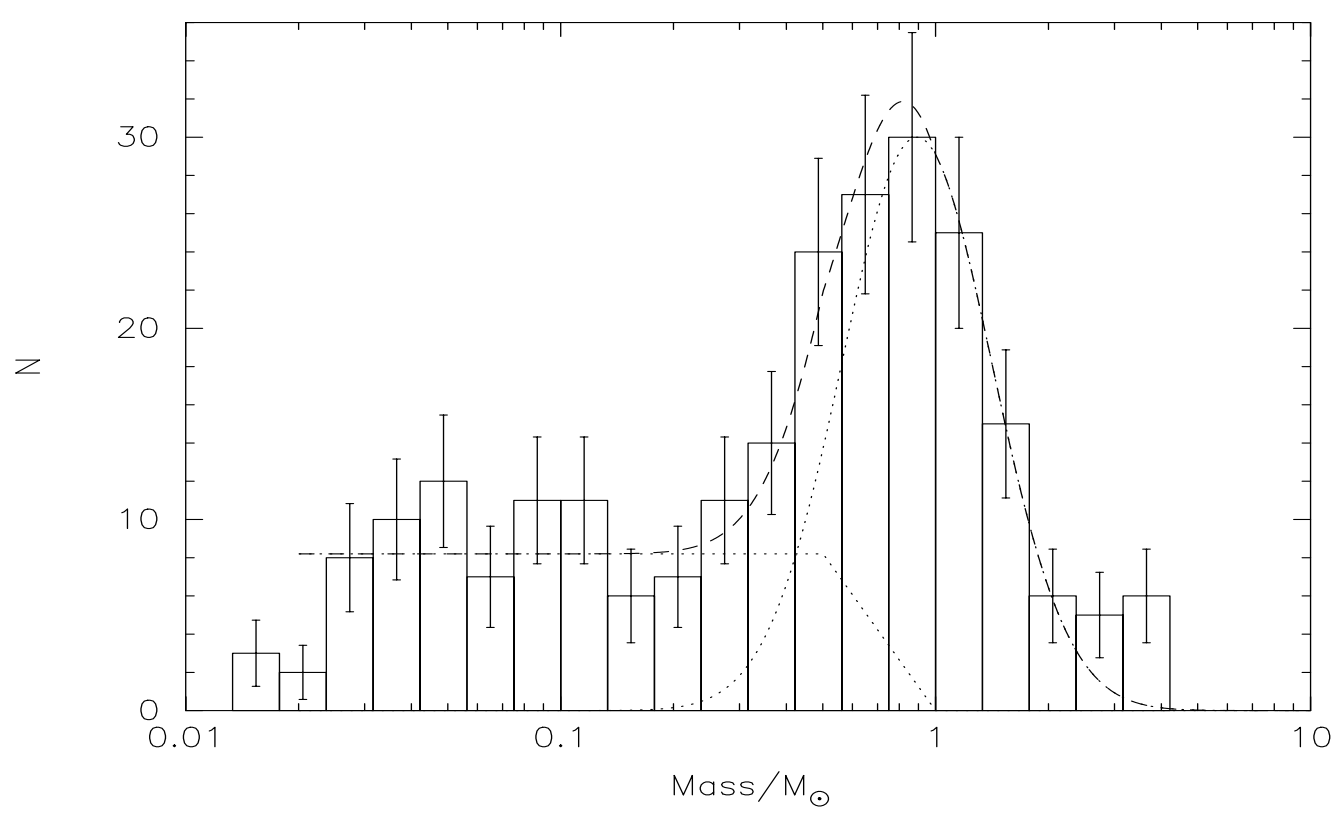

Fig. 2. The stellar IMF produced from an ensemble of fifty core collapse simulations is shown as a solid-line histogram. This histogram is well-fitted by the sum (dashed line) of two distributions (dotted lines): a log-normal distribution with mean $\log _{10}[M]=-0.05$ and standard deviation $\sigma_{\log _{10}[M]}=0.02$ plus a flat distribution below $0.5 M_{\odot}$ The stars making up the log-normal distribution generally tend to remain in multiple systems, whilst those making up the flat distribution are generally objects that have been ejected from multiple systems.

kernel softened using $h$. If the density of particle $i$ rises above $100 \rho_{\text {crit }}$, and the particles within 5 AU of particle $i$ are gravitationally bound, they are replaced with a sink particle of radius 5 AU (Bate et al. 1995).

Most simulations are made with $\mathcal{N}_{\text {tot }}=25000$ particles, but some have been repeated with $\mathcal{N}_{\text {tot }}=50000$ particles in order to test convergence. In no case is there a significant difference in the evolution using larger $\mathcal{N}_{\text {tot }}$. With $\mathcal{N}_{\text {tot }}=25000$, the minimum mass which can be resolved is $M_{\text {res }} \simeq 0.01 M_{\odot}$. With the equation of state given by Eq. (2), the minimum Jeans mass is $M_{\text {min }} \simeq 0.01 M_{\odot}$. Therefore the Jeans condition (Bate \& Burkert 1997; Whitworth 1998) is satisfied.

\subsection{Results}

We have previously shown (Goodwin et al. 2004a,b) that even cores with very low levels of turbulence can fragment to form multiple systems. In the ensemble of fifty simulations of Taurus-like cores reported here, each core forms between 1 and 9 objects, with an average of 4.5 objects. Details of the individual simulations are given in Goodwin et al. (2004a,b). Typically the lower-mass objects are ejected from the core in a period of rapid dynamical evolution (cf. Sterzik \& Durisen 2002), and the higher-mass objects remain in the core and form multiple systems. The ejected objects are lower-mass because they are ejected before they can grow much by accretion (cf. Reipurth \& Clarke 2001; Bate et al. 2002; Delgado-Donate et al. 2003, 2004; Goodwin et al. 2004a,b). The objects remaining in the core are higher-mass because they continue to accrete (cf. Bonnell et al. 2001).

Figure 2 shows as a histogram the mass function of objects produced by this ensemble of runs. The histogram is well fitted by the sum (dashed line) of two distributions (dotted lines): a narrow log-normal distribution, with mean $\left\langle\log _{10}[M]\right\rangle=-0.05$ and standard deviation $\sigma_{\log _{10}[M]}=0.02$; and a flat distribution below $0.5 M_{\odot}$. The stars contributing to the log-normal distribution are mainly those that remain in the core and end up in multiple systems. The stars contributing to the flat distribution are mainly those ejected from the core.

The low-mass objects have a flat mass distribution because their ejection is a stochastic process. In any individual core there are only a few low-mass objects, so the ejection dynamics cannot be very selective about which object is ejected (i.e. ejection is only weakly dependent on mass). The mass of an ejected object depends mainly on how long after formation it is ejected. If ejection occurs early, the object has had little time to grow and is likely to be a brown dwarf. If ejection occurs later, the object may have grown to become a low-mass star.

\section{Comparison of simulations with observations}

Figure 3 shows a comparison between the observed IMF of Taurus from Fig. 1 (solid line histogram) and the model IMF from Fig. 2, normalised and rebinned to the same binning as the observations (shaded histogram). The two histograms are very similar. A Kolmogorov-Smirnov test estimates the probability that the underlying distributions are different at less than $10 \%$. A $\chi^{2}$ test accepts the hypothesis that they are the same at more than $90 \%$ confidence.

The main discrepancy between the histograms is at high masses, where the simulations appear to produce too many stars above $1 M_{\odot}$. This may be due to the simulations not including feedback. Bipolar outflows from young stars would reduce accretion onto the more massive stars, and might thereby bring the histograms into better agreement. We are currently repeating a subset of the simulations with a more sophisticated 


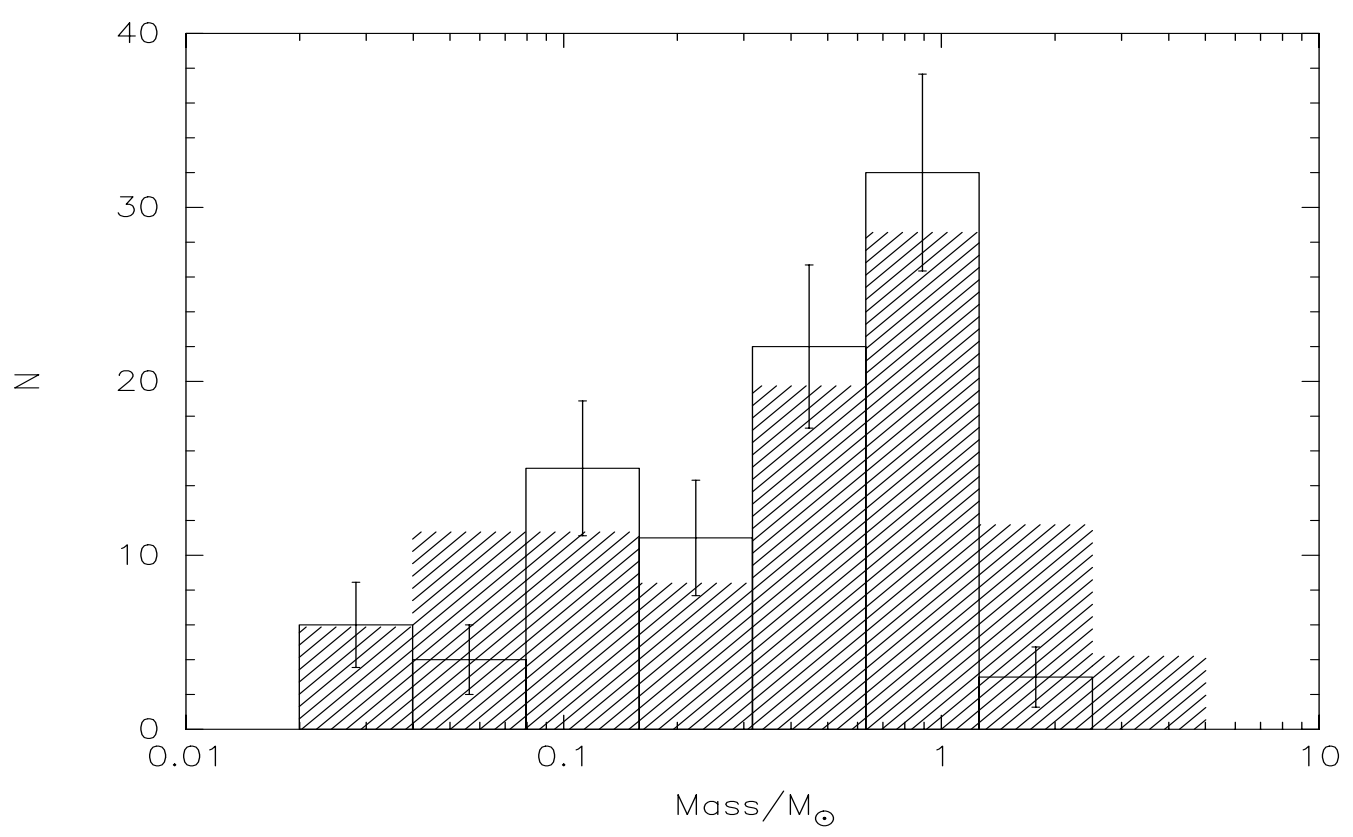

Fig. 3. The solid-line histogram shows the observed IMF of Taurus due to Luhman et al. (2003a) (as in Fig. 1). The shaded histogram shows the model IMF from Fig. 2, normalised and rebinned to match the binning of Luhman et al. (2003a). The model IMF was produced from an ensemble of simulations which starts with the observed core mass distribution in Taurus and initial conditions for individual cores (density profile, level of turbulence, etc.) based on the physical conditions observed in pre-stellar cores in Taurus. The two histograms have been normalised by minimising $\chi^{2}$. Agreement between the model and the observations is good (see text for discussion).

sink model which includes feedback due to bipolar outflows (Boyd et al., in preparation), in order to test this hypothesis.

Briceño et al. (2002) report that the spatial distributions of stars and brown dwarfs in Taurus are very similar, and this would appear to be evidence against the hypothesis that brown dwarfs are formed by ejection from star forming cores (e.g. Reipurth \& Clarke 2001; see also Kroupa \& Bouvier 2003, for a discussion in the specific context of Taurus). A similar situation is seen in Chamaeleon I by López Martí et al. (2004).

However, in our simulations the ejected objects comprise both brown dwarfs and low-mass stars, with almost twice as many low-mass stars $\left(0.08 M_{\odot}<M<0.5 M_{\odot}\right)$ as brown dwarfs $\left(M<0.08 M_{\odot}\right)$. Moreover, the ejection speed is almost independent of mass $\left(\sim 1-2 \mathrm{~km} \mathrm{~s}^{-1}\right)$, and therefore the distributions and kinematics of brown dwarfs and low-mass stars are very similar, as observed.

Moreover, any systematic difference between the velocity dispersions of objects of different mass is unlikely to produce significant segregation on timescales less than $10 \mathrm{Myr}$, for the following reasons. First, the star-forming cores in Taurus are widely spaced, and so there is no single centre from which to measure a putative diaspora of brown dwarfs or low-mass stars. Second, the individual star-forming cores have different velocities; from Onishi et al. (2002), the inter-core velocity dispersion is $\sim \pm 1 \mathrm{~km} \mathrm{~s}^{-1}$. Third, some of the older, smaller star-forming cores may already have dispersed.

\section{Conclusions}

Taurus has an unusual stellar initial mass function and an unusual core mass function. We have modelled the hydrodynamical evolution of an ensemble of cores with masses based on the
Taurus core mass function and levels of turbulence based on those observed in Taurus. We find that the unusual stellar IMF in Taurus can be explained as a direct result of the unusual core mass function and intrinsic core properties in Taurus.

In each core an initially unstable, multiple system forms, with between 2 and 9 members. Typically, 2 or 3 objects are ejected before they can accrete a significant amount of material. These ejected low-mass stars and brown dwarfs constitute the flat, low-mass "tail" of the Taurus IMF. There are almost twice as many low-mass stars $\left(0.08 M_{\odot}<M<0.5 M_{\odot}\right)$ as brown dwarfs $\left(M<0.08 M_{\odot}\right)$. The ejection velocities $\left(\sim 1-2 \mathrm{~km} \mathrm{~s}^{-1}\right)$ are essentially independent of mass, so the low-mass stars are as dispersed as the brown dwarfs and there is no significant mass segregation.

The remaining objects stay near the centre of the core and continue to accrete until their masses are $\sim 0.8 M_{\odot}$, by which stage there is not much mass left to accrete. These more massive stars constitute the Gaussian peak centred at $\sim 0.8 M_{\odot}$ in the Taurus IMF. Almost all of them are in binary or triple systems. Thus the simulated cluster of cores has an IMF very similar to that of Taurus, viz. a narrow Gaussian peak at $\sim 0.8 M_{\odot}$, and a flat tail at lower masses, extending into the brown dwarf regime.

Further support for the hypothesis that the form of the IMF in Taurus is a direct result of the typical masses of the cores in Taurus comes from Goodwin et al. (in preparation) who find that a core with low levels of turbulence typically forms a number of objects approximately equal to the number of initial Jeans masses in the core (the initial Jeans mass being roughly $\left.1 M_{\odot}\right)$. Thus lower-mass cores form fewer objects and are unable to populate the tail of the IMF as they do not eject significant numbers of brown dwarfs and low-mass stars. 
Higher-mass cores over-populate the tail through the ejection of more brown dwarfs and low-mass stars. In addition the stars that remain within a core have a larger resovoir of gas to accrete and become larger than the observed $0.8 M_{\odot}$ peak.

The agreement between the two histograms of Fig. 3 (observation and theory) is remarkably close. Hence we believe we have shown, for the first time, a direct causal link between the core mass function of a star-forming region and the stellar IMF produced in that region.

Acknowledgements. Thanks to the referee, Andi Burkert, for his useful comments. S.P.G. acknowledges support of PPARC grant $\mathrm{PPA} / \mathrm{G} / \mathrm{S} / 1998 / 00623$ and is now a UKAFF Fellow.

\section{References}

André, P., Ward-Thompson, D., \& Barsony, M. 2000, Protostars and Planets IV, ed. V. Mannings, A. P. Boss, \& S. S. Russell (Tucson: University of Arizona Press), 59

Barnes, J., \& Hut, P. 1986, Nature, 324, 446

Bate, M. R., \& Burkert, A. 1997, MNRAS, 288, 1060

Bate, M. R., Bonnell, I. A., \& Price, N. M. 1995, MNRAS, 277, 362

Bate, M. R., Bonnell, I. A., \& Bromm, V. 2002, MNRAS, 332, L65

Bonnell, I. A., Clarke, C. J., Bate, M. R., \& Pringle, J. E. 2001, MNRAS, 324, 573

Briceño, C., Luhman, K. L., Hartmann, L., Stauffer, J. R., \& Kirkpatrick, J. D. 2002, ApJ, 580, 317

Delgardo-Donate, E., Clarke, C. J., \& Bate, M. R. 2003, MNRAS, 342,926

Delgardo-Donate, E., Clarke, C. J., \& Bate, M. R. 2004, MNRAS, 347,759

Duchêne, G. 1999, A\&A, 341, 547

Ghez, A. M. 2001, The Formation of Binary Stars, ed. H. Zinnecker, \& R. D. Mathieu, IAU Symp., 200, 210

Gingold, R. A., \& Monaghan, J. J. 1977, MNRAS, 181, 375

Goodwin, S. P., Whitworth, A. P., \& Ward-Thompson, D. 2004a, A\&A, 414, 633
Goodwin, S. P., Whitworth, A. P., \& Ward-Thompson, D. 2004b, A\&A, submitted

Jijina, J., Myers, P. C., \& Adams, F. C. 1999, ApJS, 125, 161

Kirk, J. M., Ward-Thompson, D., \& André, P. 2003, MNRAS, submitted

Kroupa, P. 2002, Science, 295, 82

Kroupa, P., \& Bouvier, J. 2003, MNRAS, 346, 343

Lattanzio, J. C., Monaghan, J. J., Pongracic, H., \& Schwarz, M. P. 1986, SIAM J. Sci. Stat. Comput., 7, 591

López Martí, B., Eislöffel, J., Scholz, A., \& Mundt, R. 2004, A\&A, 416,555

Lucy, L. B. 1977, AJ, 82, 1013

Luhman, K. L., Briceño, C., Stauffer, J. R., et al. 2003a, ApJ, 590, 348

Luhman, K. L., Stauffer, J. R., Muench, A. A., et al. 2003b, ApJ, 593, 1093

Masunaga, H., \& Inutsuka, S. 2000, ApJ, 531, 350

Miller, G. E., \& Scalo, J. M. 1979, ApJSS, 41, 513

Monaghan, J. J. 1992, ARA\&A, 30, 543

Monaghan, J. J., \& Lattanzio, J. C. 1985, A\&A, 149, 135

Motte, F., André, P., \& Neri, R. 1998, A\&A, 336, 150

Motte, F., André, P., Ward-Thompson, D., \& Bontemps, S. 2001, A\&A, 372, L41

Muench, A. A., Lada, E. A., Lada, C. J., \& Alves, J. 2002, ApJ, 573, 366

Onishi, T., Mizuno, A., Kawamura, A., Tachihara, K., \& Fukui, Y. 2002, ApJ, 575, 950

Reipurth, B., \& Clarke, C. J. 2001, AJ, 122, 432

Salpeter, E. E. 1955, ApJ, 121, 161

Scalo, J. M. 1986, Fund. Cosm. Phys., 11, 1

Sterzik, M. F., \& Durisen, R. H. 2003, A\&A, 400, 1031

Testi, L., \& Sargent, A. 1998, ApJ, 508, L91

Tohline, J. E. 1982, Fund. Cosm. Phys., 8, 1

Turner, J. A., Chapman, S. J., Bhattal, A. S., et al. 1995, MNRAS, 277, 705

Ward-Thompson, D., Scott, P. F., Hills, R. E., \& André, P. 1994, MNRAS, 268, 276

Ward-Thompson, D., Motte, F., \& André, P. 1999, MNRAS, 305, 143

Ward-Thompson, D., André, P., \& Kirk, J. M. 2002, MNRAS, 329, 257

Whitworth, A. P. 1998, MNRAS, 296, 442 